\title{
Inhibition of Gli leads to antitumor growth and enhancement of cisplatin-induced cytotoxicity in large cell neuroendocrine carcinoma of the lung
}

\author{
TSUKASA ISHIWATA ${ }^{1,2}$, SHUNICHIRO IWASAWA ${ }^{1,2}$, TAKAHIRO EBATA ${ }^{2}$, MENGMENG FAN $^{2}$, \\ YUJI TADA $^{1}$, KOICHIRO TATSUMI ${ }^{1}$ and YUICHI TAKIGUCHI ${ }^{2}$ \\ Departments of ${ }^{1}$ Respirology and ${ }^{2}$ Medical Oncology, Graduate School of Medicine, \\ Chiba University, Chuo-ku, Chiba 260-8670, Japan
}

Received September 3, 2017; Accepted December 29, 2017

DOI: 10.3892/or.2018.6183

\begin{abstract}
Large cell neuroendocrine carcinoma (LCNEC) of the lung is a highly aggressive tumor without established standard treatment. The Hedgehog (Hh) signal, which is critical in embryogenesis, is known to play important roles in maintaining a malignant phenotype in various cancers. The present study explored the possibility of targeting the Hh signal in the treatment of LCNEC by suppressing Hh downstream molecules, Smoothened (Smo) and GLI family zinc finger $1 / 2$ (Gli1/2), in 3 human LCNEC cell lines. Smo inhibitor, BMS-833923, and Gli inhibitor, GANT61, downregulated Gli1 and 2, resulting in the suppression of the cell viability of the 3 cell lines as assessed using an MTT assay. The downregulation of Gli1 and/or Gli2 using siRNA for each gene also led to cell growth inhibition in the 3 cell lines. The downregulation of Gli1/2 made the cells more sensitive to cisplatin, resulting in increased apoptosis. These findings suggest that the Hh signaling pathway may be a candidate target for the treatment of LCNEC of the lung.
\end{abstract}

\section{Introduction}

The Hedgehog (Hh) signaling pathway is an evolutionarily conserved developmental pathway occurring during embryonic development that regulates cell proliferation and differentiation $(1,2)$. In the absence of extracellular Hh ligands, Patched $(\mathrm{PTCH})$ inhibits the activity of Smoothened (Smo). Signal transduction is activated when the Hh ligand binds to PTCH, relieving its inhibition of Smo, and uninhibited Smo transmits Hh signals to the nucleus through the activation of the Gli family of transcription factors. The Gli family consists of Gli1, Gli2 and Gli3; Gli1 induces Hh-target genes, Gli2 acts

Correspondence to: Professor Yuichi Takiguchi, Department of Medical Oncology, Graduate School of Medicine, Chiba University, 1-8-1 Inohana, Chuo-ku, Chiba 260-8670, Japan

E-mail: takiguchi@faculty.chiba-u.jp

Key words: Hedgehog pathway, large cell neuroendocrine carcinoma, Gli, Smo, chemosensitivity as an activator or a partial repressor, Gli3 mainly acts as a repressor (3-7). The Gli1 and Gli2 mRNA levels are relevant indicators of $\mathrm{Hh}$ pathway activity. Other than during embryonic development, the Hh pathway is also activated in mammalian adult tissues, such as the brain and bladder $(8,9)$, where it contributes to the maintenance of tissue homeostasis and facilitates tissue repair (10). Moreover, the aberrant augmented activation of $\mathrm{Hh}$ signaling has recently been shown to play a causative or promoting role in the development and progression of various human malignancies, such as lung cancer $(11,12)$, pleural mesothelioma (13), colon cancer (14), melanoma (14) and neuroblastoma (15).

Large cell neuroendocrine carcinoma (LCNEC) of the lung is a highly aggressive tumor (16-18). Although patients with early-stage disease are treated surgically, a standard therapeutic regimen for advanced-stage disease has not been established, leading to a poor therapeutic outcome. Thus, new approaches that target LCNEC are urgently needed. The Hh signaling pathway plays an important role in the growth and maintenance of a malignant phenotype in small cell lung cancer, which also has neuroendocrine features $(19,20)$. However, the roles of the Hh pathway in LCNEC remain unconfirmed. The objective of the present study was to determine whether the Hh pathway is activated in LCNEC cells and whether it contributes to cell proliferation. In addition, the potential of the Hh pathway as a therapeutic target for LCNEC of the lung was investigated.

\section{Materials and methods}

Reagents. The Smo antagonists GDC-0449 (Selleckchem, Houston, TX, USA) and BMS-833923 (Selleckchem) and the Gli antagonist GANT61 (Wako Pure Chemical Industries Ltd., Osaka, Japan) were dissolved in dimethyl sulfoxide (DMSO) in aliquots of $10 \mathrm{mM}$ stock solution and stored at $-20^{\circ} \mathrm{C}$. Cisplatin was purchased from Wako Pure Chemical Industries and was dissolved in DMSO in aliquots of $10 \mathrm{mM}$ stock solution and stored at $4^{\circ} \mathrm{C}$.

Cell lines and cell culture. Three cell lines derived from human LCNEC cells (H460, H1299 and H810) and one cell line derived from human lung adenocarcinoma cells (A549) 
were obtained from the American Type Culture Collection (ATCC, Manassas, VA, USA). H460, H1299 and A549 cells were cultured in RPMI-1640 medium (Sigma-Aldrich, St. Louis, MO, USA) supplemented with $10 \%$ fetal bovine serum (FBS). H810 cells were cultured in HITES medium with $5 \%$ FBS that contained DMEM/F12, insulin $(5 \mu \mathrm{g} / \mathrm{ml})$, transferrin $(10 \mu \mathrm{g} / \mathrm{ml})$, sodium selenite $(30 \mathrm{nM})$, hydrocortisone $(10 \mathrm{nM})$, $\beta$-estradiol $(10 \mathrm{nM})$, and L-glutamine $(4.5 \mathrm{mM})$. All the cells were cultured in a $37^{\circ} \mathrm{C}$ humidified atmosphere containing $5 \%$ $\mathrm{CO}_{2}$. According to the ATCC database, $\mathrm{H} 1299$ was confirmed as an LCNEC cell line. A previous report revealed that $\mathrm{H} 460$ and $\mathrm{H} 810$ cells express N-CAM and neuroendocrine markers such as synaptophysin or chromogranin A (21). Moreover, another report revealed that $\mathrm{H} 460$ and $\mathrm{H} 810$ cells express a high level of neuron-specific enolase (NSE) (22). The cell lines of H460, H1299 and H810 were used in several studies as LCNEC (23-25). We used A549 in this study as the most adequate positive cellular model of the Hh pathway in lung cancer. A549 cells have a higher level of expression of $\mathrm{Hh}$ pathway components than other lung cancer cell lines such as A427, NER51 or INER37 (26). Furthermore, inhibition of the Hh pathway has revealed an antitumor effect on A549 in some previous reports $(15,27)$.

Evaluation of cell viability. Cell viability was determined using the MTT dye reduction method. A total of 5,000 cells/well were seeded in 96-well culture plates. The cells were treated with various concentrations of GDC-0449, BMS-833923 or GANT61 for $48 \mathrm{~h}$. To each well, $15 \mu \mathrm{l}$ of dye solution (cat. no. G402A; Promega, Madison, WI, USA) was added, and the cells were further incubated at $37^{\circ} \mathrm{C}$ for $4 \mathrm{~h}$, followed by the addition of $100 \mu \mathrm{l}$ of stop solution (cat. no. G4001; Promega) and an additional $1 \mathrm{~h}$ of incubation. The absorbance at $570 \mathrm{~nm}$ of the resulting solution was measured using Infinite 200 PRO (FPRO-T; Tecan, Seestrasse, Switzerland). Cell viability was determined by dividing the absorbance value of the treated cells by that of the untreated cells.

Small interfering RNA (siRNA) transfection. Predesigned siRNAs targeting human Gli1 and Gli2 were purchased from Thermo Fisher Scientific (Waltham, MA, USA). As a non-specific control siRNA, scrambled siRNA duplex (Thermo Fisher Scientific) was used. Transfections were performed using Lipofectamine RNAiMAX Transfection reagent (Thermo Fisher Scientific) according to the manufacturer's instructions. Gli1 and Gli2 were silenced by siRNA for $48 \mathrm{~h}$ prior to assay or treatment.

Quantitative real-time PCR ( $q R T-P C R)$. The expression of mRNA was quantified using RT-PCR with the TaqMan Gene Expression assays, Step One Plus Real-Time PCR system, and Step One Software (Thermo Fisher Scientific). After culturing cells at $80 \%$ confluence in 6 -well culture plates, the total RNA was extracted using the RNeasy Mini kit (cat. no. 74104; Qiagen, Venlo, Limburg, The Netherlands) and the cDNA was immediately synthesized using SuperScript First-Strand Synthesis for RT-PCR (cat. no. 11904-018; Thermo Fisher Scientific) for RT-PCR according to the manufacturer's instructions. The gene expression was quantified relative to the glyceraldehyde-3-phosphate dehydrogenase (GAPDH) level.

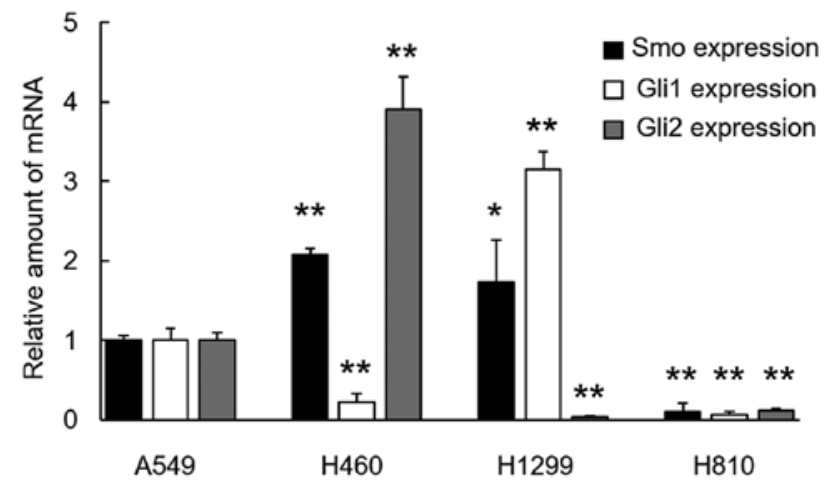

Figure 1. Expression of Hh pathway signaling components in LCNEC cell lines. The mRNA expression of Hh pathway components was evaluated in 3 human LCNEC cell lines and a human adenocarcinoma lung cell line, A549. mRNA expression of Smo, Gli1, and Gli2 assessed using qRT-PCR in the 3 cell lines was normalized according to the expression level in the A549 cells. Compared with the A549 cells, the H460 and H1299 cell lines showed a significant overexpression of Smo. H460 and H1299 cell lines also had significant overexpression of Gli2 and Gli1, respectively. In contrast, H810 had low expression levels of all 3 genes. The columns and bars represent the means and SEs $(n=3)$, respectively. Statistically significant differences, ${ }^{*} \mathrm{P}<0.05$ and ${ }^{* *} \mathrm{P}<0.01$

TaqMan probes for GAPDH, Smo, Gli1 and Gli2 were obtained by ordering from Thermo Fisher Scientific (assay identification nos. Hs02758991_g1, Hs01090242_m1, Hs00171790_m1 and Hs01119974_m1).

Apoptosis assay. To determine caspase 3 and 7 activities, the cells were seeded in 96-well plates in triplicate. At $48 \mathrm{~h}$ after treatment with cisplatin, the caspase 3 and 7 activities were determined using a Caspase-Glo 3/7 kit (cat. no. G8090; Promega), which measures caspase 3 and 7 levels in a single assay, according to the manufacturer's instructions. For the fluorescent immunohistochemical evaluation of apoptotic cells, we used the DeadEnd ${ }^{\mathrm{TM}}$ Fluorometric TUNEL system (Promega) according to the manufacturer's instructions. A total of $1 \times 10^{5}$ cells in $1 \mathrm{ml}$ of medium was distributed in each well of a Lab-Tek II Chamber Slide (Thermo Fisher Scientific). The cells were cultured overnight and treated with $10 \mu \mathrm{M}$ of cisplatin for $48 \mathrm{~h}$. A small drop of DAPI $(1 \mu \mathrm{g} / \mathrm{ml})$ in Vectashield anti-fade mounting medium (Vector Labs, Peterborough, UK) was placed on each slide, and the specimens were covered with a coverslip. The number of fluorescent-positive cells was counted using the cell counter plugin for ImageJ software (National Institutes of Health; http://imagej.nih.gov/ij/).

Statistical analyses and ethical considerations. The data are presented as the means \pm standard errors, and differences between groups were evaluated using the Student's t-test. Values of $\mathrm{P}<0.05$ (2-tailed) were considered statistically significant. All the experiments were conducted in close adherence to institutional regulations.

\section{Results}

Expression of Hh pathway signaling components. As markers for Hh signal activation, the expression levels of Gli1, Gli2 and Smo were evaluated using qRT-PCR. Gli2 and Smo in H460 cells and Gli1 and Smo in H1299 cells were overexpressed, 
A

$$
\text { A }
$$$$
\text { fH|l| : }\left\|_{1}:\right\| \|_{11}
$$

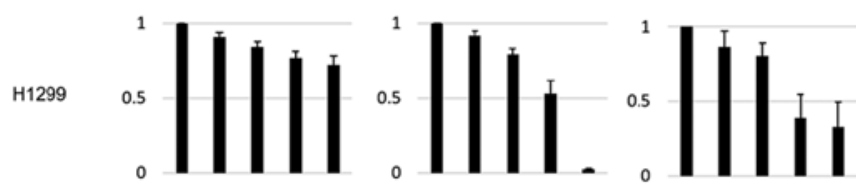$$
\mathrm{H} 810
$$

$\begin{array}{lllll}0 & 2 & 4 & 8 & 16\end{array}$

GDC0449

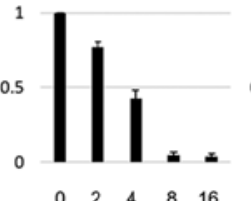

BMS833923

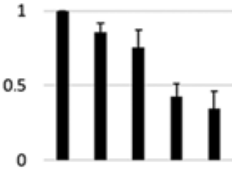

$\begin{array}{lllllll}0 & 5 & 10 & 50 & 100 & (\mu \mathrm{M})\end{array}$

GANT61
B
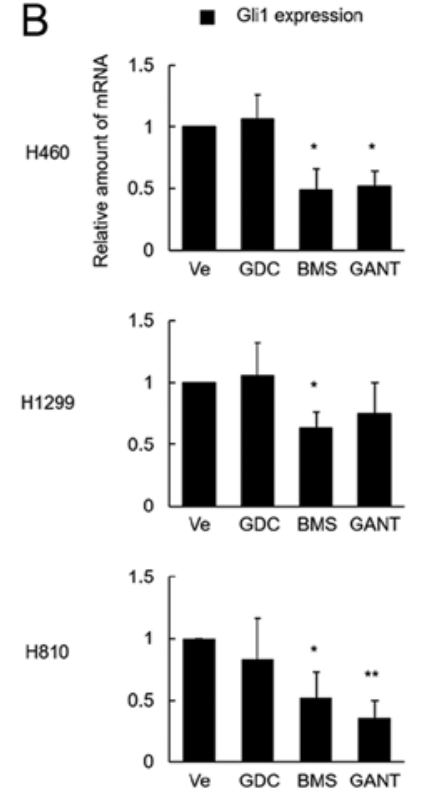

口 Gli2 expression
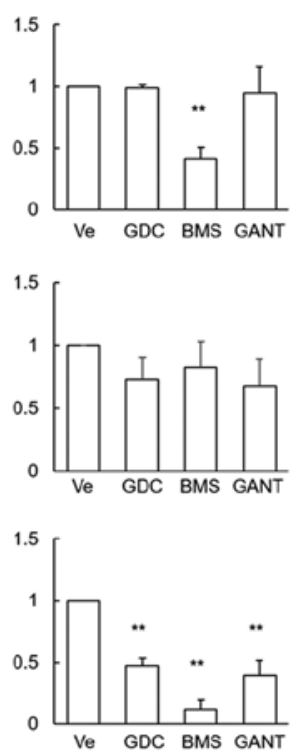

Figure 2. Effects of Smo and Gli inhibitors on LCNEC cell lines. (A) H460, H1299 and H810 cell lines were treated with various concentrations of Smo inhibitors GDC-0449 and BMS-833923 and the Gli antagonist GANT61 for 48 h. GDC-0449 exhibited limited cytotoxicity in all 3 cell lines, whereas BMS-833923 exhibited significant effects in all 3 cell lines. GANT61 was not effective enough in suppressing cell viability, as a high concentration of the agent up to $100 \mu \mathrm{M}$ was required to achieve cell viability supression. The columns and bars represent the means and SEs ( $\mathrm{n}=3$ ), respectively. (B) Downregulation of Gli1 and Gli2 mRNA by the 3 inhibitors, normalized by mRNA expression levels in the untreated cells, are shown. In this experiment, the concentrations of the 3 agents were determined according to the cell viability experiment shown in (A) and were $10 \mu \mathrm{M}$ for GDC-0449 (GDC), $5 \mu \mathrm{M}$ for BMS-833923 (BMS), and $50 \mu \mathrm{M}$ for GANT61 (GANT). Except for the expression of Gli2 mRNA in the H810 cells, GDC did not reduce the mRNA expression levels. BMS exerted significant effects on both Gli1 and Gli2 mRNA, except for the expression of Gli2 mRNA in H1299 cells. GANT suppressed Gli1 mRNA in the H460 and H810 cell lines and Gli2 mRNA in the H810 cell line. The columns and bars represent the means and SEs ( $\mathrm{n}=3$ ), respectively. Ve represents the vehicle alone, without the addition of any agents. Statistically significant differences, ${ }^{*} \mathrm{P}<0.05$ and ${ }^{* *} \mathrm{P}<0.01$.

compared with the level in the A549 cells. None of the transcription factors were overexpressed in the H810 cells (Fig. 1).

Suppression of Hh pathway signaling and cell viability by Smo or Gli antagonists. A Smo antagonist, GDC-0449, failed to exert cytotoxicity in the 3 cell lines, whereas another Smo antagonist, BMS-833923, exerted significant cytotoxicity in all 3 cell lines in a dose-dependent manner, being more pronounced in the $\mathrm{H} 460$ and $\mathrm{H} 810$ cells than in the $\mathrm{H} 1299$ cells (Fig. 2A). GDC-0449 at a concentration of $10 \mu \mathrm{M}$ failed to suppress Gli1 expression in all 3 cell lines, while it suppressed Gli2 expression solely in H810 cells. In contrast, BMS-833923 at a concentration of $5 \mu \mathrm{M}$ (this dose was determined based on the cytotoxicity effect shown in Fig. 2A) did suppress Gli1 expression in all 3 cell lines and Gli2 expression in the H460 and H810 cell lines (Fig. 2B). A Gli antagonist, GANT61, failed to show significant cytotoxicity in all 3 cell lines, except for within a high dose range of 50-100 $\mu \mathrm{M}$ (Fig. 2A). When administered at a dose as high as $50 \mu \mathrm{M}$, this agent suppressed Gli1 expression in $\mathrm{H} 460$ and $\mathrm{H} 810$ cells, whereas it suppressed Gli2 expression solely in the H810 cells (Fig. 2B).

Cytotoxicity of the silencing of Gli using siRNA. The treatment of each cell line with siRNA for Gli1 and Gli2 successfully downregulated the expression of Gli1 and Gli2, respectively. A combination of both siRNAs also successfully downregulated both factors in all 3 cell lines (Fig. 3).

The downregulation of Gli1 and Gli2 equally and significantly suppressed cell proliferation in all 3 cell lines. Although
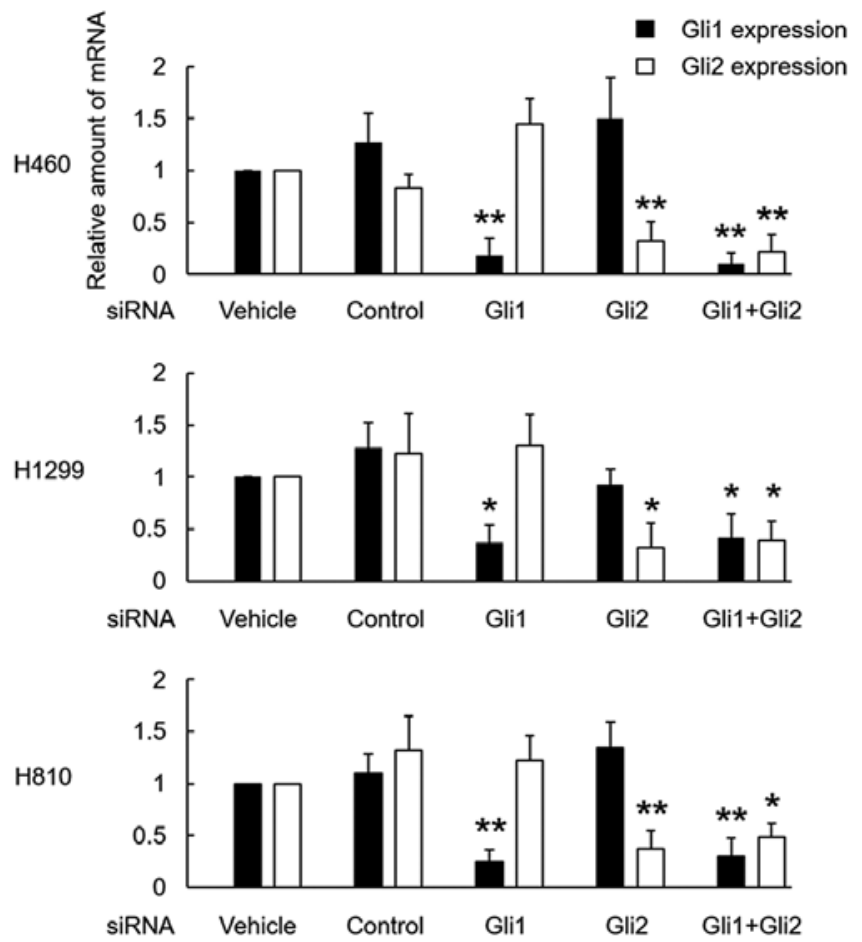

Figure 3. Silencing of Gli factors using siRNA. Each siRNA for Gli1 and Gli2 significantly inhibited the expression of the corresponding gene, and their combination significantly inhibited both genes in all 3 cell lines. In the columns for the vehicle, the cells were not treated with any siRNA, and in the columns for the control, the cells were treated with a scrambled siRNA duplex. The columns and bars represent the means and SEs $(n=3)$, respectively. Statistically significant differences are indicated by asterisks; ${ }^{*} \mathrm{P}<0.05$ and ${ }^{* *} \mathrm{P}<0.01$. 
$\mathrm{H} 460$

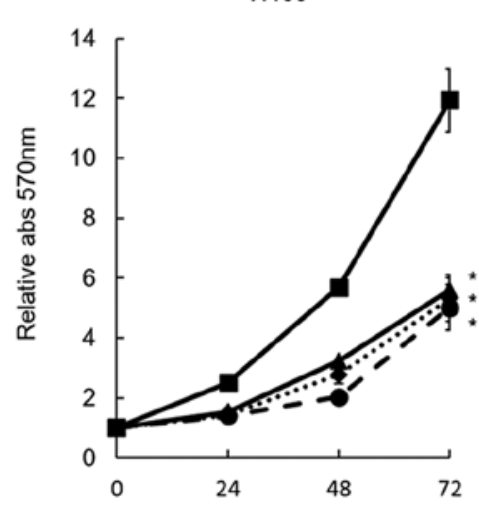

H1299

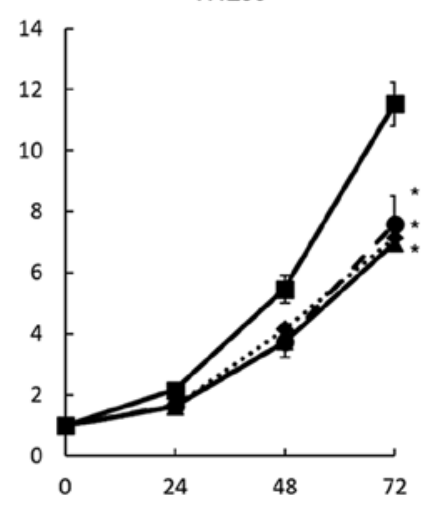

$\mathrm{H} 810$

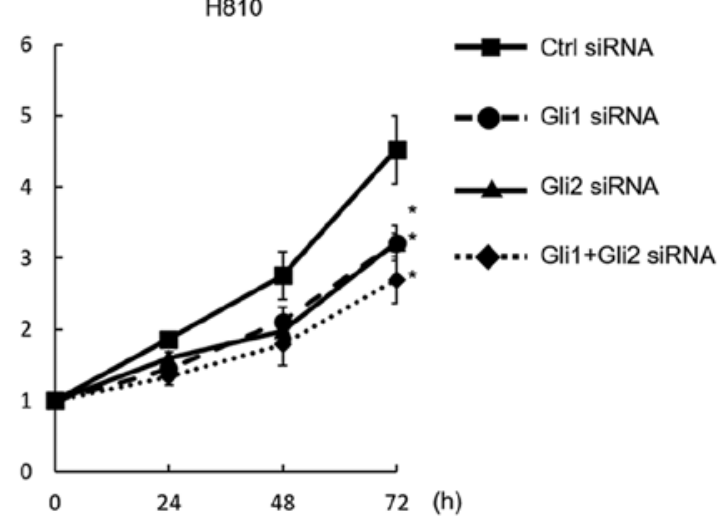

Figure 4. Effects of Gli siRNA on cell growth in LCNEC cell lines. After treatment with siRNAs for Gli1 and/or Gli2, the cells were seeded on culture plates to examine cell growth. Cell viability was determined using the MTT dye reduction method after 24,48 and 72 h. Cell growth, when treated with each siRNA for Gli1 or Gli2, was significantly inhibited ( $\mathrm{P}<0.05)$ at $72 \mathrm{~h}$, compared with the control (treatment with scrambled siRNA duplex), in all 3 cell lines. However, no additional effect was noted when these 2 siRNAs were combined. The dots and bars represent the means and SEs ( $\mathrm{n}=3$ ), respectively.

the combination of Gli1 and Gli2 downregulation significantly suppressed cell growth, no additive effect was observed by the combination, compared with the downregulation of a single gene (Fig. 4).

Cisplatin sensitivity and downregulation of Gli expression. The MTT assay demonstrated that the downregulation of Gli1, Gli2, and a combination of Gli1 and Gli2 significantly enhanced the sensitivity to cisplatin in all 3 cell lines (Fig. 5A). When treated with cisplatin, the downregulation of Gli1 and Gli2 induced significantly higher caspase 3 and 7 activity levels, compared with the control, in all 3 cell lines (Fig. 5B). A TUNEL assay also disclosed enhanced apoptosis with cisplatin treatment after Gli downregulation in the H460 and H1299 cell lines (Fig. 5C). As repeated TUNEL assays failed to show clear TUNEL-positive cells among H810 cells, the results for the $\mathrm{H} 810$ cell line are not shown.

\section{Discussion}

The present study clearly demonstrated a close relationship between the downregulation of Gli and cell growth inhibition in 3 human LCNEC cell lines of the lung. That is, the Smo inhibitor BMS-833923 and the Gli inhibitor GANT61 significantly suppressed the expression of Gli1 and/or Gli2, leading to cell growth inhibition as assessed using an MTT assay. The downregulation of Gli1 and/or Gli2 by treatment with siRNA for each gene also led to cell growth inhibition. On the other hand, another Smo inhibitor, GDC-0449, failed to downregulate Gli1 and Gli2, except for Gli2 inhibition in H810 cells, leading to non-significant growth inhibition in the 3 cell lines. Although the reason is unknown, GDC-0449 was ineffective for inhibiting the cell growth of LCNEC cells because it failed to suppress Gli expression. The action mechanisms of GDC-0449 and GANT61 are well documented. GDC-0449 binds to the transmembrane domain of Smo protein inducing a conformational change in Smo, which results in blocking the signals of normal Hh signaling (28), while the action mechanisms of BMS-833923 has not been elucidated. On the other hand, GANT61 acts in the nucleus interfering with transcriptional factor Gli binding to DNA (29). In contrast, these chemical inhibitors non-specifically suppressed the expression of both Gli1 and Gli2, and siRNA for each gene specifically suppressed the expression of either Gli1 or Gli2. Interestingly, the suppression of either Gli1 or Gli2 was shown to be sufficient to inhibit cell growth, and an additive effect was not observed for the double downregulation of both genes. Whether Gli1, Gli2 or their combination is required for Hh-related tumorigenesis remains unclear. A previous report demonstrated that Gli1 and Gli2 act as compensation for each other in mouse models (30). In some cell lines, inhibiting only Gli1 was not enough to suppress tumor growth as Gli2 can behave like Gli1 in mice (31) and Gli2 also mediates the $\mathrm{Hh}$ signaling pathway (32). Our results also showed that mRNA of Gli2 increased when Gli1 was suppressed by siRNA and vice versa, implicating those factors act in a compensational manner. On the other hand, several studies have shown that Gli1, but not Gli2, plays a central role in mediating the oncogenic Hh signaling (33-35). Interestingly, the relationship between Hh signaling and tumor proliferation was observed even in $\mathrm{H} 810$ cells despite the absence of the overexpression of these genes in this cell line, as shown in Fig. 1. We showed that Hh inhibitors and siRNAs effectively suppressed these baseline expression levels of Gli1/2 together with suppression of cell growth (Figs. 2-4) in H810 cells. It might be speculated that Gli1/2 are essential for cell growth independent of baseline expression. Similar phenomena were also observed: Gli1 downregulation in $\mathrm{H} 460$ cells led to cell growth inhibition despite the fact that these cells did not overexpress Gli1, while Gli2 downregulation in H1299 cells led to cell growth inhibition despite the fact that these cells do not overexpress Gli2. These facts suggest that both Gli1 and Gli2 independently have critical roles in maintaining cell growth ability irrespective of their baseline expression levels. In a previous report regarding malignant pleural mesothelioma cells, a single treatment with Gli1 or Gli2 siRNA did not produce significant inhibitory effects on cell growth, whereas the double downregulation of Gli1 and Gli2 significantly inhibited cell proliferation (36). Therefore, the independence of Gli1 and Gli2 might vary among tumor types.

The present study also demonstrated a close relationship between Gli downregulation and an increased sensitivity 
A H460

H1299 H810
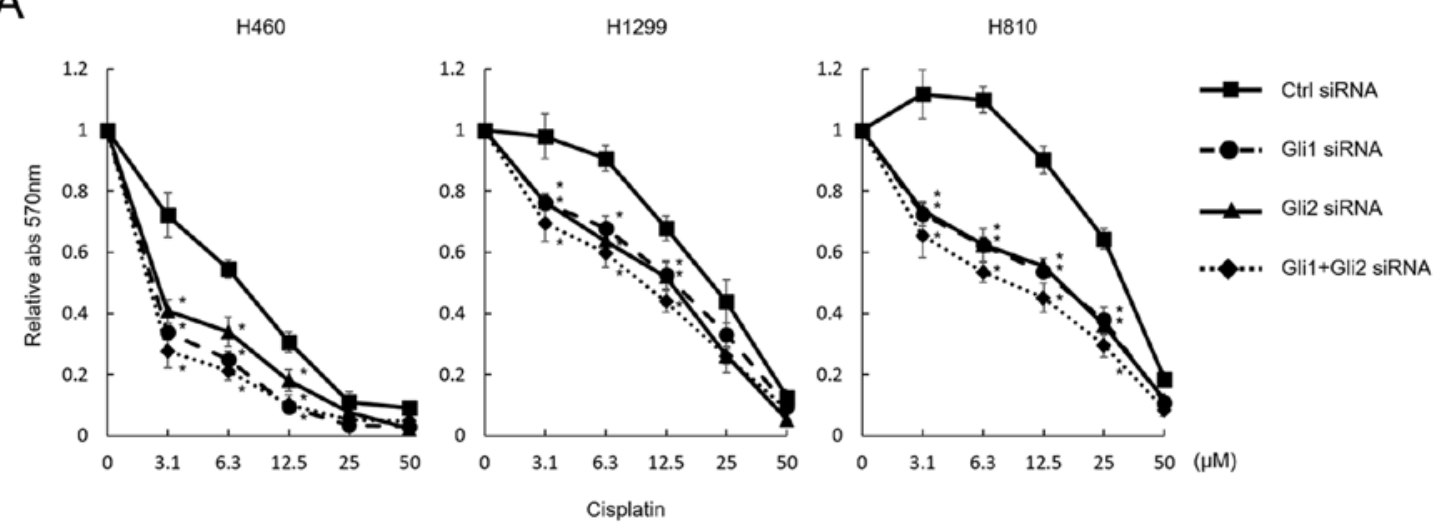

B
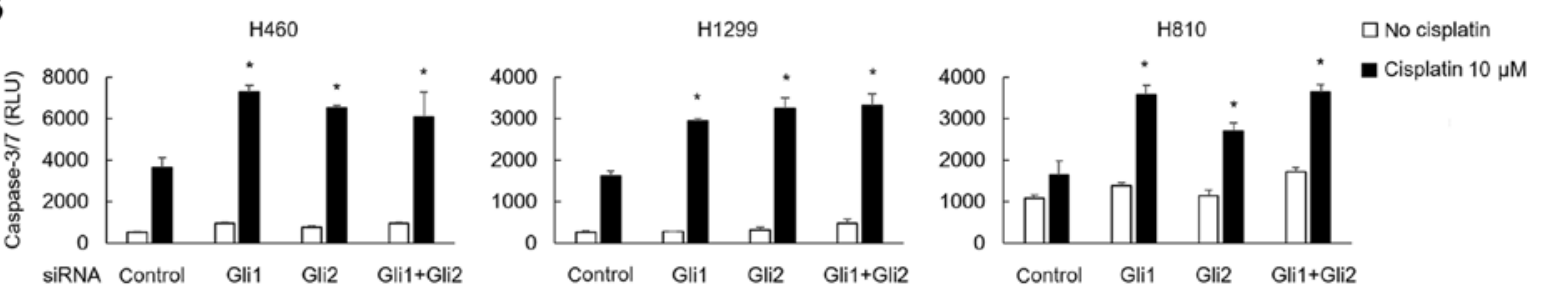

C

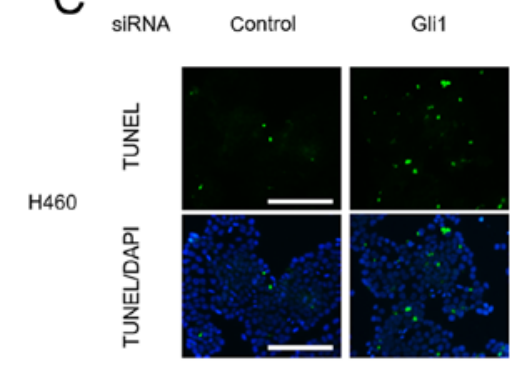

Gli2

Gli1+Gli2
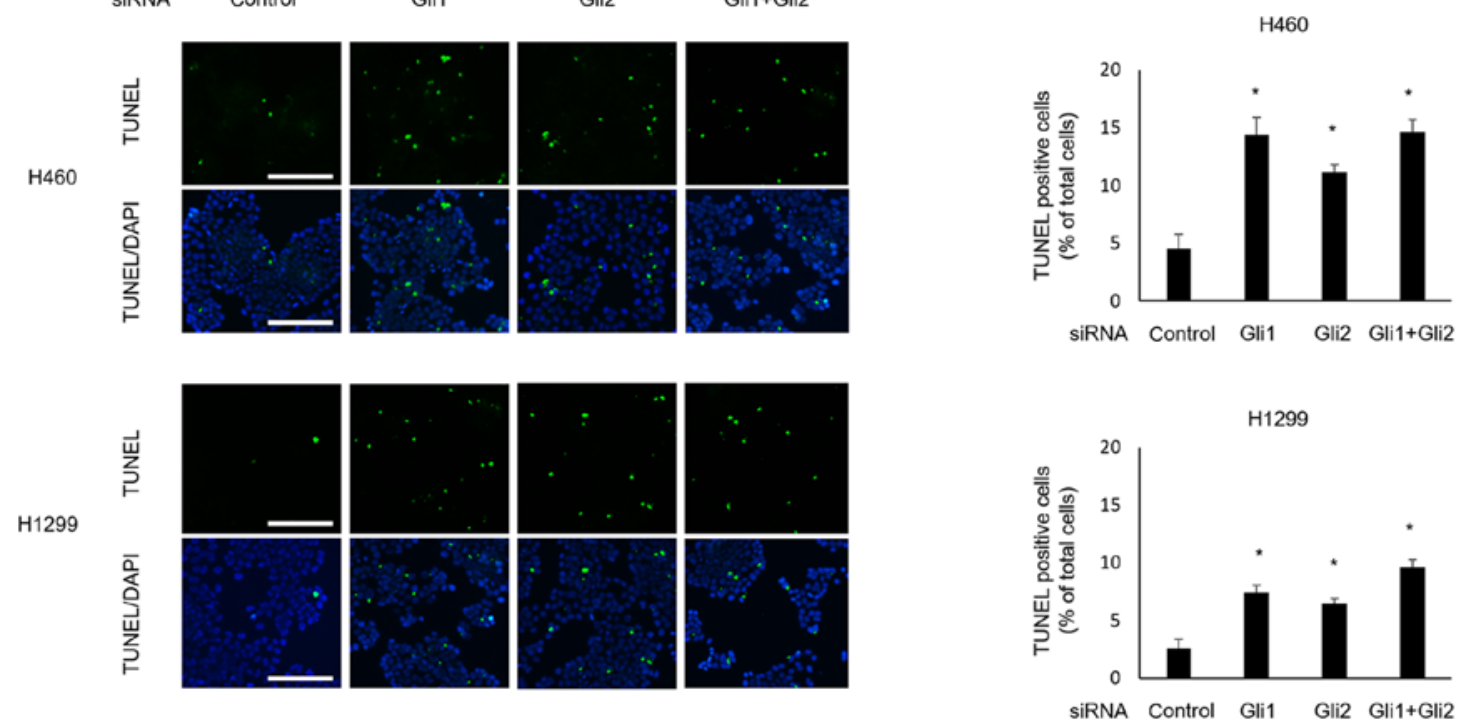

Figure 5. Cisplatin sensitivity and downregulation of Gli expression. (A) After treatment with siRNA, the cells were next treated with various concentrations of cisplatin for $48 \mathrm{~h}$. Treatment with siRNA for Gli1, Gli2, or both made all 3 cell lines significantly ("P<0.05) more sensitive to cisplatin. To verify this phenomenon, induction of apoptosis was evaluated using a caspase 3/7 assay (B) and a TUNEL assay (C and D). (B) Caspase 3/7 activity was assessed at $48 \mathrm{~h}$ post-treatment with cisplatin at $10 \mu \mathrm{M}$, and the activity was significantly enhanced in cells treated with siRNA for Gli1, Gli2, or both, whereas apoptosis was not induced in cells treated without cisplatin and with siRNA treatment alone. Again, no additive effect was observed for the combination of both siRNAs. (C) Apoptotic cells after treatment with cisplatin were evaluated and visualized using a TUNEL assay. H460 and H1299 cells treated with Gli1 and/or Gli2 siRNA were further treated with $10 \mu \mathrm{M}$ of cisplatin for $48 \mathrm{~h}$. The green and blue fluorescence of DAPI shows apoptotic cells and nuclei, respectively. Scale bar, $100 \mu \mathrm{m}$. (D) The proportions of apoptotic cells were quantified for comparison among the treatment groups. The number of TUNEL-positive cells was significantly higher after treatment with siRNA for Gli1 and/or Gli2. Statistically significant differences, relative to the control ("P<0.05) in (A), (B) and (D). The columns and bars represent the means and SEs $(n=3)$, respectively, in (B) and (D).

to cisplatin. That is, the downregulation of Gli1 and/or Gli2 made the cells more sensitive to cisplatin, possibly through an increase in apoptosis as was assessed using caspase $3 / 7$ and TUNEL assays. A previous study also revealed that Gli2 knockdown using an antisense oligonucleotide led to enhanced chemosensitivity to paclitaxel in prostate cancer (37). Another study showed that treatment with GANT61 enhanced the cytotoxicity of cytarabine for acute myeloid leukemia (38). As to the underlying mechanism, Sims-Mourtada et al reported that Gli1 regulates the ATP-binding cassette transporter family 
of proteins that is required for drug efflux (39). Amable et al showed that Gli1 plays a role in the cellular accumulation of cisplatin through the regulation of multiple transport proteins including octamer-binding protein (OCT)1, OCT2, OCT3, the copper transporter CTR1 and the ATPase copper transporter ATP7B (40). On the other hand, cisplatin, in turn, affects the Hh pathway. After treatment with cisplatin, DNA repair mechanisms, including nucleotide excision repair, mismatch repair and DNA double strand break repair, are upregulated in cancer cells to avoid apoptosis. Upregulated Hh signaling helps the DNA repair mechanisms. Suppression of Gli1 by GANT61 downregulated the genes related to double-strand break repair by homologous recombination (41) and nucleotide excision repair (42). Therefore, there is possibility of synergistic effects of concurrent exposure to cisplatin and Gli knockdown. The shortcomings of this study contain the lack of protein expression analysis, the lack of confirmation with in vivo experiments and the lack of elucidating precise mechanism due to its preliminary nature. Despite these limitations, the present study provides evidence supporting Gli as a therapeutic target for the treatment of LCNEC of the lung, which is presently an unmet need.

In conclusion, the present study suggests that Gli activation plays a critical role in LCNEC proliferation and drug sensitivity. The inhibition of Gli factors has the potential to become an effective approach to the treatment of LCNEC of the lung.

\section{Acknowledgements}

This study was supported by grants from the Ministry of Education, Culture, Sports, Science and Technology in Japan of grant no. 26860599 to S.I., and nos. 26461182 and 17K09647 to Y.T.

\section{References}

1. Nüsslein-Volhard C and Wieschaus E: Mutations affecting segment number and polarity in Drosophila. Nature 287: 795-801, 1980.

2. Ingham PW and McMahon AP: Hedgehog signaling in animal development: Paradigms and principles. Genes Dev 15: 3059-3087, 2001.

3. Chen JK, Taipale J, Cooper MK and Beachy PA: Inhibition of Hedgehog signaling by direct binding of cyclopamine to Smoothened. Genes Dev 16: 2743-2748, 2002.

4. Duman-Scheel M, Weng L, Xin S and Du W: Hedgehog regulates cell growth and proliferation by inducing Cyclin D and Cyclin E. Nature 417: 299-304, 2002

5. Lum L and Beachy PA: The Hedgehog response network: Sensors, switches, and routers. Science 304: 1755-1759, 2004

6. Taipale J, Chen JK, Cooper MK, Wang B, Mann RK, Milenkovic L, Scott MP and Beachy PA: Effects of oncogenic mutations in Smoothened and Patched can be reversed by cyclopamine. Nature 406: 1005-1009, 2000.

7. Sasaki H, Nishizaki Y, Hui C, Nakafuku M and Kondoh H: Regulation of Gli2 and Gli3 activities by an amino-terminal repression domain: Implication of Gli2 and Gli3 as primary mediators of Shh signaling. Development 126: 3915-3924, 1999.

8. Alvarez JI, Dodelet-Devillers A, Kebir H, Ifergan I, Fabre PJ, Terouz S, Sabbagh M, Wosik K, Bourbonnière L, Bernard M, et al: The Hedgehog pathway promotes blood-brain barrier integrity and CNS immune quiescence. Science 334: 1727-1731, 2011.

9. Shin K, Lee J, Guo N, Kim J, Lim A, Qu L, Mysorekar IU and Beachy PA: Hedgehog/Wnt feedback supports regenerative proliferation of epithelial stem cells in bladder. Nature 472: 110-114, 2011.

10. Petrova R and Joyner AL: Roles for Hedgehog signaling in adult organ homeostasis and repair. Development 141: 3445-3457, 2014
11. Bermudez O, Hennen E, Koch I, Lindner M and Eickelberg O: Gli1 mediates lung cancer cell proliferation and Sonic Hedgehogdependent mesenchymal cell activation. PLoS One 8: e63226, 2013.

12. Huang L, Walter V, Hayes DN and Onaitis M: Hedgehog-GLI signaling inhibition suppresses tumor growth in squamous lung cancer. Clin Cancer Res 20: 1566-1575, 2014.

13. You M, Varona-Santos J, Singh S, Robbins DJ, Savaraj N and Nguyen DM: Targeting of the Hedgehog signal transduction pathway suppresses survival of malignant pleural mesothelioma cells in vitro. J Thorac Cardiovasc Surg 147: 508-516, 2014.

14. Bosco-Clément G, Zhang F, Chen Z, Zhou HM, Li H, Mikami I, Hirata T, Yagui-Beltran A, Lui N, Do HT, et al: Targeting Gli transcription activation by small molecule suppresses tumor growth. Oncogene 33: 2087-2097, 2014.

15. Wickström M, Dyberg C, Shimokawa T, Milosevic J, Baryawno N, Fuskevåg $\mathrm{OM}$, Larsson R, Kogner P, Zaphiropoulos PG and Johnsen JI: Targeting the hedgehog signal transduction pathway at the level of GLI inhibits neuroblastoma cell growth in vitro and in vivo. Int J Cancer 132: 1516-1524, 2013.

16. Rieber J, Schmitt J, Warth A, Muley T, Kappes J, Eichhorn F, Hoffmann H, Heussel CP, Welzel T, Debus J, et al: Outcome and prognostic factors of multimodal therapy for pulmonary largecell neuroendocrine carcinomas. Eur J Med Res 20: 64, 2015.

17. Niho S, Kenmotsu H, Sekine I, Ishii G, Ishikawa Y, Noguchi M, Oshita F, Watanabe S, Nakajima R, Tada H, et al: Combination chemotherapy with irinotecan and cisplatin for large-cell neuroendocrine carcinoma of the lung: A multicenter phase II study. J Thorac Oncol 8: 980-984, 2013.

18. Battafarano RJ, Fernandez FG, Ritter J, Meyers BF, Guthrie TJ, Cooper JD and Patterson GA: Large cell neuroendocrine carcinoma: An aggressive form of non-small cell lung cancer. J Thorac Cardiovasc Surg 130: 166-172, 2005.

19. Watkins DN, Berman DM, Burkholder SG, Wang B, Beachy PA and Baylin SB: Hedgehog signalling within airway epithelial progenitors and in small-cell lung cancer. Nature 422: 313-317, 2003.

20. Vestergaard J, Pedersen MW, Pedersen N, Ensinger C, Tümer Z, Tommerup N, Poulsen HS and Larsen LA: Hedgehog signaling in small-cell lung cancer: Frequent in vivo but a rare event in vitro. Lung Cancer 52: 281-290, 2006.

21. Carbone DP, Koros AM, Linnoila RI, Jewett P and Gazdar AF: Neural cell adhesion molecule expression and messenger RNA splicing patterns in lung cancer cell lines are correlated with neuroendocrine phenotype and growth morphology. Cancer Res 51: 6142-6149, 1991.

22. Senden N, Linnoila I, Timmer E, van de Velde H, Roebroek A, Van de Ven W, Broers J and Ramaekers F: Neuroendocrinespecific protein (NSP)-reticulons as independent markers for non-small cell lung cancer with neuroendocrine differentiation. An in vitro histochemical study. Histochem Cell Biol 108: 155-165, 1997.

23. Koyama N, Zhang J, Huqun, Miyazawa H, Tanaka T, Su X and Hagiwara K: Identification of IGFBP-6 as an effector of the tumor suppressor activity of SEMA3B. Oncogene 27: 6581-6589, 2008.

24. Odate S, Onishi H, Nakamura K, Kojima M, Uchiyama A, Kato $\mathrm{M}$ and Katano M: Tropomyosin-related kinase B inhibitor has potential for tumor regression and relapse prevention in pulmonary large cell neuroendocrine carcinoma. Anticancer Res 33: 3699-3703, 2013.

25. Tatematsu T, Sasaki H, Shimizu S, Okuda K, Shitara M, Hikosaka Y, Moriyama S, Yano M, Brown J and Fujii Y: Investigation of neurotrophic tyrosine kinase receptor 1 fusions and neurotrophic tyrosine kinase receptor family expression in non-small-cell lung cancer and sensitivity to AZD7451 in vitro. Mol Clin Oncol 2: 725-730, 2014.

26. Armas-López L, Zúñiga J, Arrieta O and Ávila-Moreno F: The Hedgehog-GLI pathway in embryonic development and cancer: Implications for pulmonary oncology therapy. Oncotarget 8: 60684-60703, 2017.

27. Giroux Leprieur E, Vieira T, Antoine M, Rozensztajn N, Rabbe N, Ruppert AM, Lavole A, Cadranel J and Wislez M: Sonic Hedgehog pathway activation is associated with resistance to platinum-based chemotherapy in advanced non-small-cell lung carcinoma. Clin Lung Cancer 17: 301-308, 2016.

28. Byrne EFX, Sircar R, Miller PS, Hedger G, Luchetti G, Nachtergaele S, Tully MD, Mydock-McGrane L, Covey DF, Rambo RP, et al: Structural basis of Smoothened regulation by its extracellular domains. Nature 535: 517-522, 2016. 
29. Lauth M, Bergström A, Shimokawa T and Toftgård R: Inhibition of GLI-mediated transcription and tumor cell growth by smallmolecule antagonists. Proc Natl Acad Sci USA 104: 8455-8460, 2007.

30. Regl G, Kasper M, Schnidar H, Eichberger T, Neill GW, Philpott MP, Esterbauer H, Hauser-Kronberger C, Frischauf AM and Aberger F: Activation of the BCL2 promoter in response to Hedgehog/GLI signal transduction is predominantly mediated by GLI2. Cancer Res 64: 7724-7731, 2004.

31. Bai CB and Joyner AL: Gli1 can rescue the in vivo function of Gli2. Development 128: 5161-5172, 2001.

32. Roessler E, Du YZ, Mullor JL, Casas E, Allen WP, GillessenKaesbach G, Roeder ER, Ming JE, Ruiz i Altaba A and Muenke M: Loss-of-function mutations in the human GLI2 gene are associated with pituitary anomalies and holoprosencephalylike features. Proc Natl Acad Sci USA 100: 13424-13429, 2003.

33. Regl G, Neill GW, Eichberger T, Kasper M, Ikram MS, Koller J, Hintner H, Quinn AG, Frischauf AM and Aberger F: Human GLI2 and GLI1 are part of a positive feedback mechanism in Basal Cell Carcinoma. Oncogene 21: 5529-5539, 2002.

34. Dahmane N, Lee J, Robins P, Heller P and Ruiz i Altaba A: Activation of the transcription factor Gli1 and the Sonic hedgehog signalling pathway in skin tumours. Nature 389: 876-881, 1997.

35. Bonifas JM, Pennypacker S, Chuang PT, McMahon AP, Williams M, Rosenthal A, De Sauvage FJ and Epstein EH Jr: Activation of expression of hedgehog target genes in basal cell carcinomas. J Invest Dermatol 116: 739-742, 2001.
36. Li H, Lui N, Cheng T, Tseng HH, Yue D, Giroux-Leprieur E, Do HT, Sheng Q, Jin JQ, Luh TW, et al: Gli as a novel therapeutic target in malignant pleural mesothelioma. PLoS One 8: e57346, 2013.

37. Narita S, So A, Ettinger S, Hayashi N, Muramaki M, Fazli L, Kim Y and Gleave ME: GLI2 knockdown using an antisense oligonucleotide induces apoptosis and chemosensitizes cells to paclitaxel in androgen-independent prostate cancer. Clin Cancer Res 14: 5769-5777, 2008.

38. Long B, Wang LX, Zheng FM, Lai SP, Xu DR, Hu Y, Lin DJ, Zhang XZ, Dong L, Long ZJ, et al: Targeting GLI1 suppresses cell growth and enhances chemosensitivity in CD34 $4^{+}$enriched acute myeloid leukemia progenitor cells. Cell Physiol Biochem 38: 1288-1302, 2016

39. Sims-Mourtada J, Izzo JG, Ajani J and Chao KS: Sonic Hedgehog promotes multiple drug resistance by regulation of drug transport. Oncogene 26: 5674-5679, 2007.

40. Amable L, Fain J, Gavin E and Reed E: Gli1 contributes to cellular resistance to cisplatin through altered cellular accumulation of the drug. Oncol Rep 32: 469-474, 2014.

41. Shi T, Mazumdar T, Devecchio J, Duan ZH, Agyeman A, Aziz M and Houghton JA: cDNA microarray gene expression profiling of hedgehog signaling pathway inhibition in human colon cancer cells. PLoS One 5: 5, 2010.

42. Mazumdar T, DeVecchio J, Agyeman A, Shi T and Houghton JA: The GLI genes as the molecular switch in disrupting Hedgehog signaling in colon cancer. Oncotarget 2: 638-645, 2011. 